against latitude and across-track distance. It represents the increase in total error as a percentage of distance run when the across-track distance is not changing rapidly.

If $X$ is along-track distance and $Y$ is across-track distance, total error in an east/west direction

$$
=\int_{0}^{X} Y \frac{f^{\prime}(m)}{f(m)} d X
$$

For a sphere,

$$
\frac{f^{\prime}(m)}{f(m)}=\frac{\tan \varphi}{a}
$$

where $\varphi=$ latitude and $a=$ radius of the Earth.

Therefore the approximate total error in computer position on a sphere is

$$
\frac{1}{a} \int_{0}^{X} Y \tan \phi d X
$$

This correction could be incorporated in the computer if heading references justified the requirement for accuracy.

\title{
Calculation of Charts on an Oblique Gnomonic Projection by Electronic Computer
}

\author{
from P. B. Sarson
}

(Meteorological Office)

IN the Meteorological Office, great accuracy is not usually attainable in determining or forecasting the position of significant weather features. Special projections of charts are therefore not often required; the normal conic projection with two standard parallels or (near the equator) the mercator projection is quite adequate. However, in the radiolocation of thunderstorms a chart drawn on a gnomonic projection is required. The bearings of each lightning flash within one or two thousand miles are recorded from a small number of special stations (sFerics stations). When these bearings are plotted on a gnomonic chart by straight lines drawn from the appropriate observing station the coordinates of the source of the lightning can be quickly determined and reported through normal meteorological channels. Speed is essential and therefore the charts on which the bearings are drawn are specially designed with the SFERICS stations grouped more or less evenly about the tangential point of the projection plane of the gnomonic chart.

Direction roses are marked in special borders (one for each station) round the edges of the chart so that the bearings can be plotted instantly using a length of cord drawn through a small hole in the chart, at the position of the station, to the appropriate bearing given in the direction-rose border.

Several of these charts have been calculated by pencil-and-paper methods as the demand for a chart for any particular area arose, although none covering so 


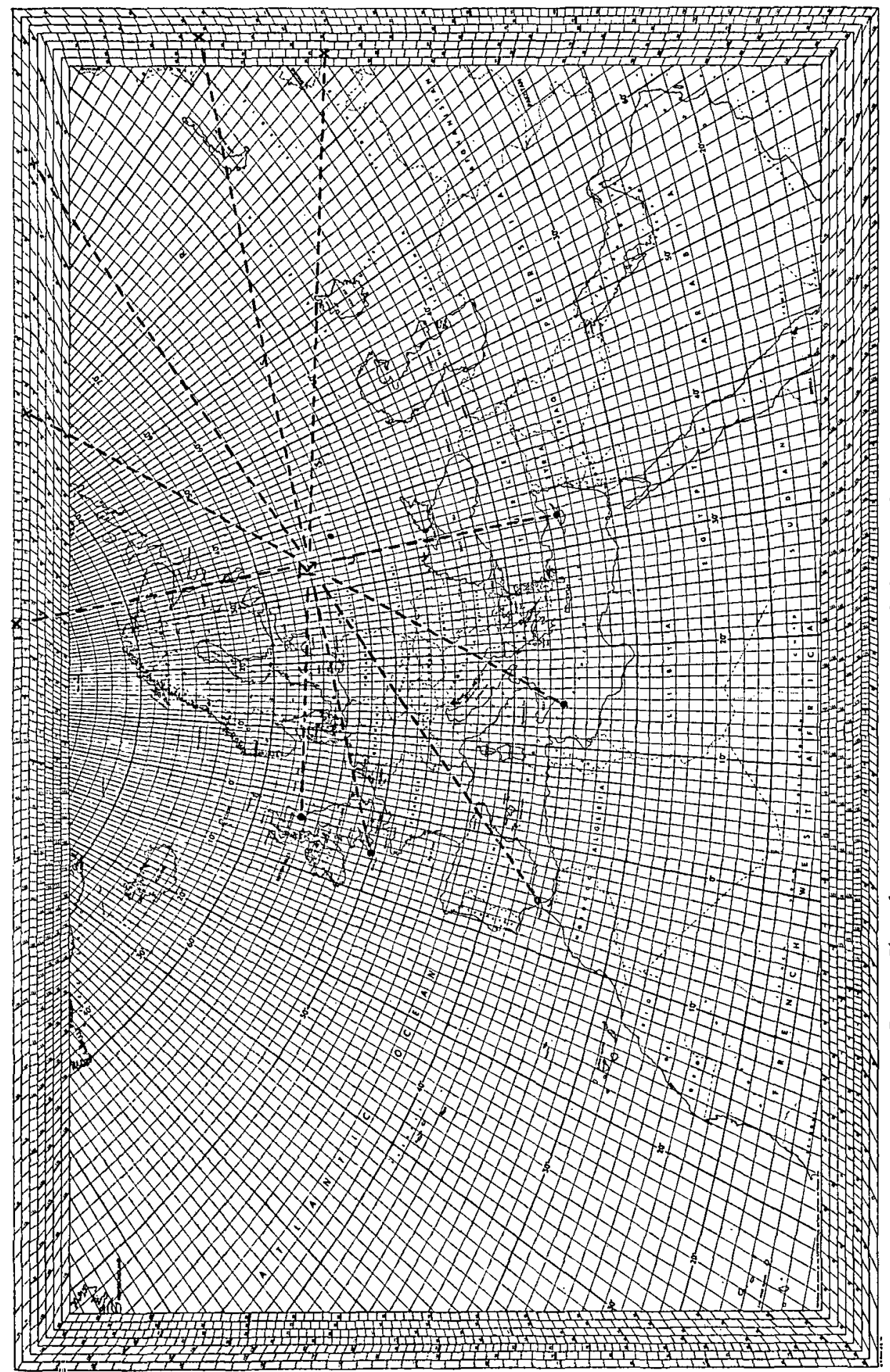

$\dot{j}$

bo

.

.

t

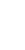

总

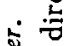

ํㅗㄴ

范

䒓苔

ह वे

造

$\therefore 8 \pi$

공

正

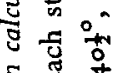

(

政

¿

ป 0

है

ธ్

0 a

5 .

ป है

인

늄

约

$\therefore \stackrel{0}{2}$

की हो

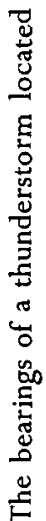


large an area as the one illustrated. With the introduction of an electronic computer into the Meteorological Office it was decided to do all the calculations on the computer, and a programme was devised which, without any effort other than the insertion of appropriate constants as data into the computer, prints out, in order:

(i) the intercepts along the borders of the map of each longitude,

(ii) the distances from the border along each longitude of each intersecting latitude,

(iii) the distances along each appropriate border of the bearings from any point within the chart whose coordinates are given.

Item (i) requires the calculation of six trigonometrical functions plus a division, one or two multiplications and a few additions and subtractions for each intercept. Item (ii) requires the calculation of two trigonometrical functions, one division, two or three multiplications, and a few additions and subtractions for each latitude. Item (iii) requires a small preliminary calculation including six trigonometrical functions, one division, twenty-eight multiplications and eight additions or subtractions (all this is accomplished within $\frac{1}{15}$ sec.), and then, for each bearing, two more trigonometrical functions, twelve multiplications, one division and eight additions or subtractions.

The slowest part of the programme is the actual printing out of the solution which is limited by the speed of the punch which transforms the answer as obtained in the machine to teleprinter tape. The output is thus limited (even printing to only four-figure accuracy or less than half that used by the machine and with the punch operating at full speed) to three numbers a second. The teleprinter on which the results are printed is five times as slow as the punch (even when working at full speed). Thus the coordinates for the chart illustrated took some 40-45 minutes to punch out, plus another i 5 minutes for the direction roses. The printed solution was obtained from the teleprinter in a little under 5 hours. With the high-speed printer now available it should be possible to keep up with the computer, and print the whole solution out in some 1 5-20 minutes. This compares with an estimated time of calculation, using logarithmic and trigonometrical tables, of about three months.

The solution is quite general as to the position of the tangential point of the projection plane, but the chart must not cover so large an area of the Earth's surface that latitudes $60^{\circ}$ south of the tangential point are included within it. The stations for which direction roses are required must lie within the borders of the chart.

This note is published by permission of the Director-General of the Meteorological Office.

\section{Detection of Gross Instrumentation}

\section{Errors}

$$
\text { from J.B. Parker }
$$

Mr. J. F. Green, in an article on gyro failure (this Journal, 13, 7.8) draws .attention to a principle that is of direct importance whenever navigators are 